\title{
Eccentricity estimates in hierarchical triple systems
}

\author{
Nikolaos Georgakarakos ${ }^{1}$ and Siegfried Eggl ${ }^{2}$ \\ ${ }^{1}$ Higher Technological Educational Institute of Central Macedonia, Serres, Greece \\ email: georgakarakos@hotmail.com \\ ${ }^{2}$ IMCCE, Observatoire de Paris, Paris, France \\ email: siegfried.eggl@imcce.fr
}

\begin{abstract}
Perturbation theory in the three body problem has greatly advanced our ability to understand and model a variety of systems ranging from artificial satellites to stars and from extrasolar planets to asteroid-Jupiter interactions. In a series of papers, we developed an analytical technique for estimating the orbital eccentricity of the inner binary in hierarchical triple systems. The method combined the secular theory with calculations of short period terms. The derivation of the short term component was based on an expansion of the rate of change of the Runge-Lenz vector by using first order perturbation theory, while canonical perturbation theory was used to investigate the secular evolution of the system. In the present work we extend the calculation to the orbit of the outer binary. At the same time, we provide an improved version for some previous results. A post-Newtonian correction is included in our model. Our analytical estimates are compared with numerical and analytical results on the subject and applications to stellar triples and extrasolar planets are discussed.
\end{abstract}

Keywords. (Stars:) binaries: general, Celestial mechanics, Planets and satellites: dynamical evolution and stability.

\section{Introduction}

In a series of papers (Georgakarakos 2002, 2003, 2004, 2006, 2009), we focused on the evolution of the inner eccentricity of a hierarchical triple system. In the present work, we derive analytical expressions for the outer eccentricity of a coplanar hierarchical triple system, with a particular interest in the case where the outer body is of planetary mass.

\section{Theory}

The derivation of the short period terms follows the same basic technique described in the papers for the inner eccentricity (differentiation and then integration of the RungeLenz vector). However, in the expressions for the two Jacobian vectors, instead of the true anomaly, we use the eccentric anomaly: $\mathbf{r}=a_{1}\left(\cos E_{1}-e_{1}, \sqrt{1-e_{1}^{2}} \sin E_{1}\right)$ and $\mathbf{R}=$ $a_{2}\left(\cos l_{2}, \sin l_{2}\right)$, where the indices 1 and 2 refer to the inner and outer orbit respectively, $\mathbf{r}$ is the relative position vector of the inner orbit, $\mathbf{R}$ is the relative position vector of the outer orbit, $a, e, E$ and $l$ denote semi-major axis, eccentricity, eccentric anomaly and mean anomaly respectively. Eventually, retaining the two dominant terms in our expressions, we get:

$$
\begin{aligned}
e_{21 s h}(t)= & \frac{m_{0} m_{1}}{\left(m_{0}+m_{1}\right)^{\frac{4}{3}} M^{\frac{2}{3}}} \frac{1}{X^{\frac{4}{3}}}\left[\frac{3}{4} \cos l_{2}+e_{1}^{2}\left(\frac{33}{16} \cos l_{2}+\frac{35}{16} \cos 3 l_{2}\right)\right. \\
& \left.+\frac{P_{1}(t)}{X}\right]+C_{e_{21 s h}}
\end{aligned}
$$




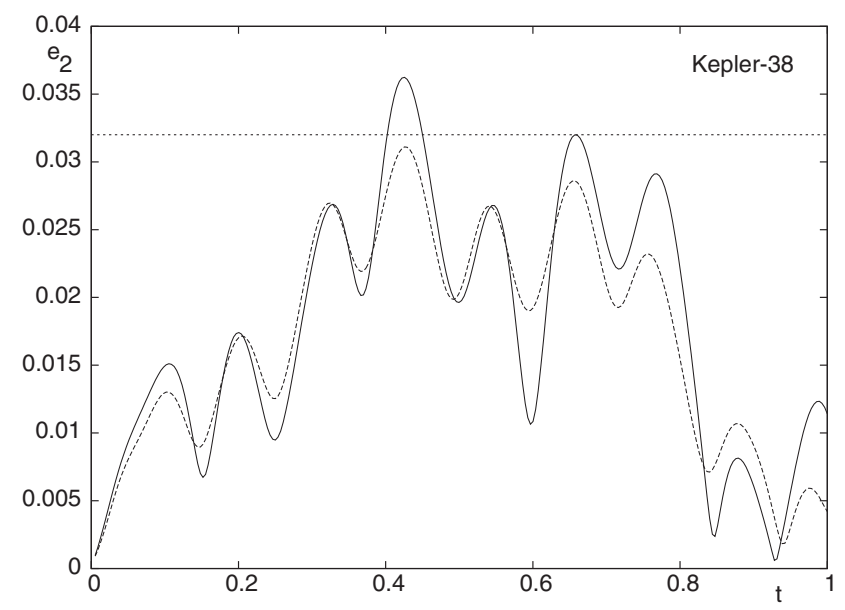

Figure 1. Short term eccentricity evolution of the circumbinary planet in the Kepler-38 system (continues line: numerical solution of the full equations of motion, dashed line: our analytical estimate). The planet was started with $e_{2}=0$ and the straight line shows its current eccentricity of 0.032 . The time is given in planetary periods.

$$
\begin{aligned}
e_{22 s h}(t)= & \frac{m_{0} m_{1}}{\left(m_{0}+m_{1}\right)^{\frac{4}{3}} M^{\frac{2}{3}}} \frac{1}{X^{\frac{4}{3}}}\left[\frac{3}{4} \sin l_{2}+e_{1}^{2}\left(\frac{3}{16} \sin l_{2}+\frac{35}{16} \sin 3 l_{2}\right)\right. \\
& \left.+\frac{P_{2}(t)}{X}\right]+C_{e_{22 s h}},
\end{aligned}
$$

where $m_{0}$ and $m_{1}$ are the masses of the inner binary, $M$ is the total mass of the system, $\mathrm{X}$ is the period ratio, $P_{1}(t)$ and $P_{2}(t)$ are lengthy expressions containing terms with frequencies of the order of the inner mean motion and $C_{e_{21 s h}}, C_{e_{22 s h}}$ are constants of integration.

\section{Applications}

Fig. 1 is an application of the above equations to the Kepler-38 system.

\section{Outlook}

The next step is to obtain expressions for the secular evolution of the system (including a post-Newtonian correction), derive estimates for the maximum and averaged eccentricity, check the range of their validity by means of numerical simulations and finally use the estimates for the determination of planetary habitable zones in stellar binaries (e.g. Eggl et al. 2012).

\section{References}

Eggl, S., Pilat-Lohinger, E., Georgakarakos, N., Gyergyovits, M., \& Funk, B. 2012, ApJ, 752, 74

Georgakarakos, N. 2002, MNRAS, 337, 559

Georgakarakos, N. 2003, MNRAS, 345, 340

Georgakarakos, N. 2004, Celes. Mech. Dyn. Astr., 89, 63

Georgakarakos, N. 2006, MNRAS, 366, 566

Georgakarakos, N. 2009, MNRAS, 392, 1253 